\title{
Coarctation of Cord: An Unidentified Cause of Fetal Demise
}

\author{
Anamika Singh ${ }^{1}$, Manjushri Waikar ${ }^{2}$
}

\begin{abstract}
Coarctation of the umbilical cord is featured as segmental or diffused absence of Wharton's jelly, resulting in the constriction of cord and narrowing of vessels. It can develop at any gestational age and is unrelated to parity. The stricture of the cord is usually associated with torsion and characterized by fibrosis of Wharton's jelly and thickening of the vessel walls, which hampers the fetoplacental circulation, resulting in hypoxia/anoxia and subsequently fetal demise. Although this event must be noticed frequently in practice, it is often not reported by the obstetricians because examination of the umbilical cord is not performed in all cases of unexplained stillbirth.

Keywords: Coarctation of cord, Cord stricture, Fetal demise, Thrombosis, Umbilical cord accidents.

International Journal of Infertility and Fetal Medicine (2019): 10.5005/jp-journals-10016-1179
\end{abstract}

\section{INTRODUCTION}

Fetal death involves a large proportion of perinatal death. However, even by the latest listing of causes using the "Extended Wigglesworth classification," it has been observed that cause of fetal death is not known in $60 \%$ of stillbirths. ${ }^{1}$

Although cord strictures and overcoiling have been shown to be the causes of intrauterine fetal demise, ${ }^{2-4}$ this topic has been addressed in only a few studies. Some authors concluded that abnormalities of these cords are an unusual cause of fetal death. In their analysis, only Machin et al. ${ }^{4}$ found that cord coiling abnormality with or without cord stricture was significantly associated with adverse perinatal results.

No large sample studies have been conducted to assess the role of cord anomalies in unexplained fetal deaths, instead most of them are case reports. ${ }^{5-7}$ But the problem with these studies is that maintaining an intact cord is very difficult and it is even harder to obtain a gestational sac with the in situ fetus and placenta held together by intact cord. Nearly $80 \%$ of abortions are observed to occur in the first trimester, and $53 \%$ of these are expected to be due to chromosomal abnormalities. ${ }^{8}$ Still in $45 \%$ of cases it is unknown.

Likewise, in a vast majority of cases, the cause of abortion in the early second trimester is unclear. Therefore, assessing the role of cord complications in unexplained intrauterine fetal death (IUFD), especially those occurring during early pregnancy is of utmost importance.

The umbilical cord coarctation is an event consisting of cord narrowing, absence of Wharton's jelly, thickening of the vessel walls and luminal narrowing, resulting in fetal hypoxia/anoxia and leading to fetal death. This narrowing may involve either whole length of cord or segments. At the fetal end of the umbilical cord, near the umbilicus, the segmental presence is normally found as a single constriction; even though, the cord may be constricted anywhere. It may occur at a single place or more than one place. Typically, the constriction is accompanied by torsion and hypercoiling.

Weber ${ }^{9}$ cited a 19th century study in 1963 (Dhorn, 1861) mentioning many clinical reports referring to dozens of cases of fetal death caused by umbilical cord constriction. Ruysch (1691) was the first to describe a case of cord torsion, but Burdach (1758) was the first to explain torsion-constriction interaction.

The pathological changes noted are inflammatory changes that may cause regressive lesions in cord, resulting in cord fibrosis.
1,2Department of Obstetrics and Gynaecology, Government Medical College, Nagpur, Maharashtra, India

Corresponding Author: Anamika Singh, Department of Obstetrics and Gynaecology, Government Medical College, Nagpur, Maharashtra, India, Phone: +91 9082208509, e-mail: dr.anamika27.AS@gmail.com

How to cite this article: Singh A, Waikar M. Coarctation of Cord: An Unidentified Cause of Fetal Demise. Int J Infertil Fetal Med 2019;10(1):12-14.

Source of support: Nil

Conflict of interest: None

Inflammatory changes in the umbilical cord are relatively common, mostly nonspecific vasculitis previously imagined to be syphilitic lesions, as per Kaufmann² (1957).

One case report ${ }^{10}$ documented the occurrence of torsion in a woman in three of her pregnancies, all of which ended in abortions, suggesting umbilical cord stricture to be a genetic anomaly. However, according to Rodriguez et al., ${ }^{11}$ while repetitive cases may imply a pathogenic mechanism that leads to recurrence, environmental and acquired aspects should be considered before genetic factors. Their research supports the notion that umbilical cord stricture is not a genetic anomaly. The recurrence found in previous studies can be explained by chance association. They also recommend that parents who have a fetal death due to cord stricture should be recommended to have a low risk of recurrence for subsequent pregnancies.

\section{Case Description}

A 24 year-old woman, G2A1, was diagnosed with IUFD at 19 weeks 4 days of gestation. The cord was twisted at the umbilicus' insertion point. Growth restriction and anatomic abnormalities were absent. Her pregnancy progressed without complications until 19 weeks when she observed the absence of fetal movements for 2 days. She did not have any history of trauma, vaginal bleeding, or pain in the abdomen. No fetal cardiac activity with reduced liquor was confirmed on ultrasound examination. No growth lag or fetal anatomic anomalies were appreciated. No history of leaking per vaginum was given by patient. After counseling of parents and taking the written consent patient was induced. A macerated 


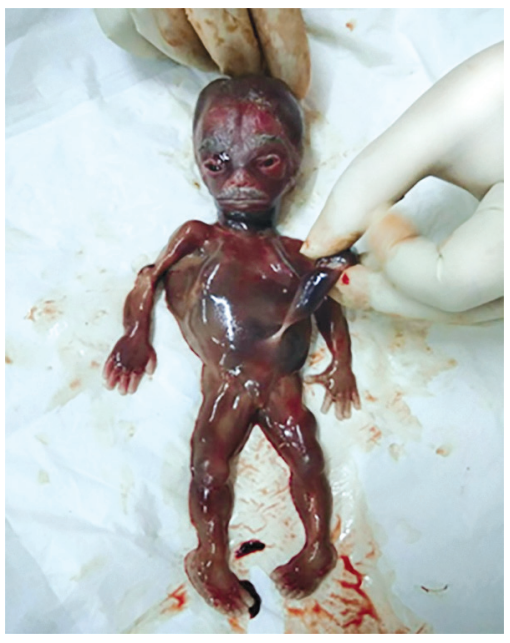

Fig. 1: Fetal demise demonstrating gross view of umbilical cord stricture near fetal end

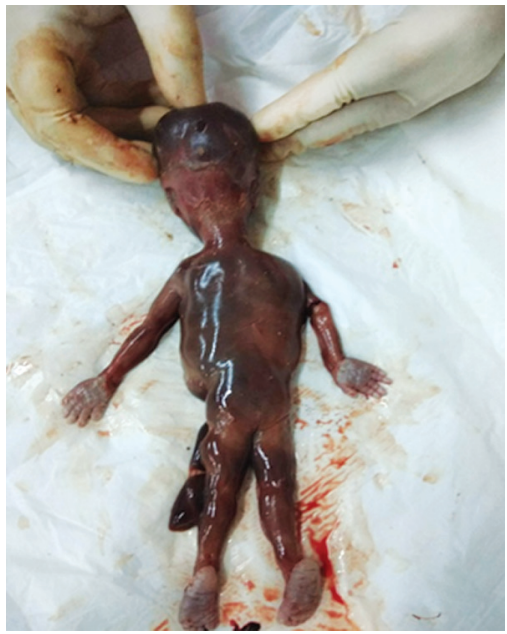

Fig. 2: Anatomically normal fetus

female dead fetus of $260 \mathrm{~g}$ was delivered vaginally. There have been no gross anatomic abnormalities found. No signs of placental infarction were noted. Cord was observed to be twisted at the fetal insertion site and was congested (Figs 1 and 2). The karyotype of the fetus was $46, X X$. The fetal death was assumed to be due to the umbilical cord stricture.

\section{Discussion}

Wharton's jelly is the connective tissue recognized as a protective surface to secure the umbilical vessels. Localized defects in Wharton's jelly production causes weakness in the cord around which the fetus can rotate to induce torsion. The location of torsion is usually close to the fetus. Edema usually occurs distally to the area or areas affected (many strictures can occur). It reduces blood flow through the area of the stricture and may have fatal consequences for the developing fetus. Coarctation of the umbilical cord shows no racial bias and is unrelated to the gestational period, parity, or sex of the fetus.

A case of umbilical cord stricture was also reported in a 21 week fetus by Wahl et al. ${ }^{12}$ Several other studies in literature showed the correlation between constriction and fetal death.,13,14 It is caused due to prolonged ischemia superimposed by an acute vascular event.
Hypercoiling can also be associated with constriction and long cords. The hypercoiled cord is often thin and the torsion is focal at times. Hypercoiling has been shown by several studies to contribute to fetal death. ${ }^{4,6,15}$ While analyzing the correlation between cord coiling and perinatal outcomes, Machin et al. ${ }^{4}$ found that overcoiled cords were associated with a high incidence of fetal demise, growth delay, and fetal labor intolerance making them susceptible to distress.

Diagnosing the stricture is very difficult because it cannot be seen using ultrasound. A reduction in fetal movements is the only symptom observed. Diagnosis of coarctation of the cord is impossible in asymptomatic pregnancies. It may be assumed after fetal demise, especially if it is preceded by irregular and vigorous fetal movements followed by their cessation. This pattern of fetal behavior can occur in any circumstance that causes sudden fetal hypoxia interrupting fetoplacental circulation and causes sudden fetal anoxia. Coarctation may also start at an early stage of cord development, with fetal death from sudden anoxia resulting in severe impedance in fetoplacental circulation. Impairment in fetoplacental circulation may be aggravated by twisting cord, seen to occur after a sudden fetal change of position. Therefore, confirmatory diagnosis can be made postdelivery only when localized cord strictures (usually accompanied by torsion at the same level) can be seen via naked eye. Hence, the umbilical cord needs to be evaluated carefully in each and every case of IUFD. These tests can reveal the cause of so many IUFDs that are usually considered unexplainable.

Grossly, many fetuses are macerated and an extremely narrow segment of the umbilical cord is usually seen at the fetal end and rarely at the placental end or at multiple sites along the cord. The major pathological characteristics are absence of Wharton's jelly, stenosis, or obliteration of cord vessels and thrombosis of cord. ${ }^{14}$

\section{Conclusion}

We agree that umbilical cord coarctation occurs frequently, but is often ignored because in all cases of IUFD the umbilical cord is not carefully examined. This omission may explain why the most authorities failed to mention the coarctation of the umbilical cord. The mechanism of cord stricture and how it results in fetal death remains unknown. If the exact etiology of cord stricture can be determined, efforts can be directed to prevent recurrence. It may be helpful for the same to have additional published cases and prospective studies.

\section{References}

1. Wigglesworth JS, Singer DB, ed. Textbook of Fetal and Perinatal Pathology, 2nd ed. Malden, MA: Blackwell; 1998. pp. 84-85.

2. Benirschke K, Kaufmann P. Pathology of the Human Placenta, 3rd ed. New York: Springer-Verlag; 1995. p. 332.

3. Sun Y, Arbuckle S, Hocking G. Umbilical cord stricture and intrauterine fetal death. Pediatr Path Lab Med 1995;15(5):723-732. DOI: 10.3109/15513819509027008.

4. Machin GA, Ackerman J, Gilbert-Barness E. Abnormal umbilical cord coiling is associated with adverse perinatal outcomes. Pediatr Dev Pathol 2000;3(5):462-471. DOI: 10.1007/s100240010103.

5. Gilbert EF, Zugibe FT. Torsion and constriction of the umbilical cord. A case of fetal death. Arch Pathol 1974;97(1):58-59.

6. Glanfield PA, Watson R. Intrauterine fetal death due to umbilical cord torsion. Arch Pathol Lab Med 1986;110(4):357-358.

7. King EL. Intrauterine death of the fetus due to abnormalities of the umbilical cord: report of three cases. Am J Obstet Gynecol 1926;12(6):812-816. DOI: 10.1016/S0002-9378(15) 32517-5. 
8. Cunningham FG, Macdonald PC, et al. Abortion. In: Williams Obstetrics, 20th ed., Stamford, Conn: Appleton \& Lange; 1997. pp. 579-605.

9. Weber J. Constriction of the umbilical cord as a cause of the fetal death. Acta Obstet Gynecol Scand 1963;42(3):259. DOI: 10.3109/00016346309158112.

10. Bakotic BW, Boyd T, Poppiti R, et al. Recurrent umbilical cord torsion leading to fetal death in 3 subsequent pregnancies: a case report and review of the literature. Arch Pathol Lab Med 2000;124:1352-1355.

11. Rodríguez Jl, Mariño-Enríquez A, Suárez-Aguado J, et al. Umbilical Cord Stricture is Not a Genetic Anomaly: A Study in Twins. Pediatr Dev Pathol 2008;11(5):363-369. DOI: 10.2350/07-08-0329.1.
12. Wahl C, Masliah E. Umbilical cord stricture in a 21-week fetus. J Perinatol 2004;24(1):48-49. DOI: 10.1038/sj.jp.7211015.

13. Hallak M, Pryde PG, Qureshi F, et al. Constriction of the umbilical cord leading to fetal death: a report of three cases. J Reprod Med 1994;39(7):561-565.

14. Virgilio LA, Spangler DB. Fetal death secondary to constriction and torsion of the umbilical cord. Arch Pathol Lab Med 1978;102(1): 32-33.

15. Herman $A$, Zabow $P$, Segal $M$, et al. Extremely large number of twists of the umbilical cord causing torsion and intrauterine fetal death. Int J Gynecol Obstet 1991;35(2):165-167. DOI: 10.1016/0020-7292(91) 90821-L. 